\title{
Lecturas que abrigan, voces que acogen. Implementación de un taller de lectura con niños de la comunidad de Sacsamarca en el marco de la Responsabilidad Social Universitaria (RSU)
}

Sección ESTUDIOS

RECIBIDO: $21 / 10 / 2020$

APROBADO: 10/11/2021

PUBLICADO ONLINE: 30/12/2021

\section{Carmen María Sandoval Figueroa}

Pontificia Universidad Católica del Perú Grupo de investigación LIED-CISE sandoval.cm@pucp.pe

https://orcid.org/0000-0003-3239-4158

\section{RESUMEN}

Durante el año 2019, una docente y un grupo de estudiantes de un programa de formación de docentes de una universidad privada de Lima diseñaron un taller de lectura dirigido a niños de la comunidad rural de Sacsamarca (Ayacucho), las edades de los menores oscilaron entre 3 y 12 años. Los objetivos del taller fueron generar un espacio de lecturas compartidas para que los niños tengan acceso al libro fuera del contexto escolar e implementar un sistema de préstamos de libros a domicilio para promover la circulación de los libros. El taller fue parte del proyecto Como Jugando, con enfoque de responsabilidad social universitaria (RSU), tuvo una duración de 12 sesiones ( 2 por mes) entre junio y diciembre. El taller de lectura fue de propuesta permanente, coexistiendo con otras estaciones con propuestas educativas como eljuego al aire libre, arte creativo, música, psicomotricidad, entre otras. El taller tuvo como sustento metodológico el enfoque de la mediación de lectura. El diseño de los talleres, así como su ejecución estuvieron a cargo de los estudiantes. Las propuestas de lecturas fueron diversas, dando respuesta a los intereses y propuestas de los niños. Finalmente, esta experiencia muestra que los niños se interesan por la lectura y se apropian de ella, motivando interacciones entre lectores a partir de los momentos de las lecturas en voz alta y la conversación.

PALABRAS CLAVE: infancia, educación alternativa, formación de docentes, lectura.

\section{Readings that shelter, voices that welcome. Implementation of a reading workshop with children from the community of Sacsamarca within the framework of the University Social Responsibility(USR)}

\section{ABSTRACT}

In 2019, a teacher and a group of students from a teacher training program at a private university in Lima designed a reading workshop aimed at children from the rural community of Sacsamarca

(c) Los autores. Este artículo es publicado por la Revista de Sociología de la Facultad de Ciencias Sociales, Universidad Nacional Mayor de San Marcos. Este es un artículo de acceso abierto, distribuido bajo los términos de la licencia Creative Commons Atribución 4.0 Internacional (CC BY 4.0) [https://creativecommons.org/licenses/by/4.0/deed.es] que permite el uso, distribución y reproducción en cualquier medio, siempre que la obra original sea debidamente citada de su fuente original. 
(Ayacucho) whose ages ranged from 3 to 12 years. The objectives of the workshop were to create a space for shared reading so that children have access to the book outside of the school context and to implement a home loan system for books to promote the circulation of books. The workshop was part of the "Como Jugando" project, with a focus on university social responsibility and had a duration of 12 sessions ( 2 per month) between June and December. The reading workshop was a permanent proposal, coexisting with other educational proposals such as outdoor play, creative art, music, psychomotor skills, among others. The workshop's methodological support was the approach of reading mediation. The design of the workshops, as well as their execution, were in charge of the students. The reading proposals were diverse, responding to the interests and proposals of the children. Finally, this experience has shown children interested in reading and appropriation of content, motivating interactions between readers at reading out loud and conversation moments.

KEYWORDS: childhood, alternative education, teacher education, reading.

\section{Introducción}

I voluntariado se inició en respuesta a una convocatoria de la Dirección Académica de Responsabilidad Social (a partir de ahora DARS) de la universidad e incluyó a estudiantes de dos especialidades de la Facultad de Educación, Inicial y Primaria. En total fueron 9 estudiantes y una docente del Departamento de Educación que integraron este voluntariado. La experiencia de los talleres fue generadora de vínculo entre los estudiantes, dado que la planificación, ejecución y evaluación de los talleres les permitió trabajar en grupo, coordinar y tomar decisiones, acciones que les permitió consolidarse como equipo y contribuyó a dar sostenibilidad de los talleres. Además, los voluntarios contaron con el apoyo de una docente asesora así como del acompañamiento formativo del equipo de la DARS' Esta experiencia colaboró al logro de competencias en los estudiantes: el trabajo en equipo, aprendizaje autónomo y comunicación eficaz.

Asimismo, los estudiantes en coordinación con la docente asesora presentaron la experiencia en el V Encuentro Nacional "Responsabilidad Social: Fundamento de la Vida Universitaria" Ilevado a cabo en la ciudad de Ayacucho el 14 y 15 de noviembre del 2019. De otra parte, la experiencia ha sido presentada por la docente asesora en el XVI Congreso Latinoamericano para el Desarrollo de la Lectura y la Escritura, organizado virtualmente por el Comité Latinoamericano para el Desarrollo de la Lectura y la Escritura (CLA), el Consejo Puebla de Lectura A. C. (CPL) y la Universidad Iberoamericana de Puebla (IBERO Puebla) del lunes 23 al viernes 27 de agosto de 2021. Con la finalidad de sistematizar la experiencia para este artículo se consulta-

1 El acompañamiento formativo hace referencia a una acción que facilita la generación de confianza entre las comunidades y la PUCP. Se trata de una relación que se construye de forma horizontal y basada en la confianza mutua, la empatía y el cuidado (Del Castillo, 2018). 
ron las programaciones de talleres y se realizó una entrevista grupal con 6 de los estudiantes voluntarios en agosto del año 2020.

Antes de continuar explicando sobre el taller de lectura, se considera importante hacer una referencia a Sacsamarca, la comunidad donde se realizaron los talleres y que se encuentra ubicada en el departamento de Ayacucho. Es un distrito de la provincia de Huancasancos que se encuentra en la zona central del departamento. El centro poblado de Sacsamarca es la capital del distrito que está conformada por los anexos de Colcabamba, Pallca, Asca y Putacassa (Espinoza, Escudero, Mamani et al., 2018). Los talleres que se realizaron con los niños se ejecutaron en el centro poblado, específicamente en las instalaciones de la municipalidad que está ubicada en la Plaza de Armas. En Sacsamarca los pobladores hablan el quechua y el español, pero en espacios cotidianos hablan sobre todo quechua.

El vínculo colaborativo que se estableció entre la comunidad de Sacsamarca y la universidad se genera en el año 2013 , se establece un convenio a solicitud de las autoridades de Sacsamarca y la PUCP, donde se promueve el trabajo colaborativo que da como resultado el convenio. Uno de los objetivos fue "Fortalecer el desarrollo de una ciudadanía democrática que reconoce la diversidad para la construcción de proyectos comunes en Sacsamarca" (Protocolo DARS docentes, 2018, p. 1). Otro propósito fue "contribuir al bienestar de los hombres y mujeres de este pueblo golpeado por el conflicto armado interno durante los años ochenta" (Espinoza, Escudero, Mamani et al., 2018, p. 19).

Cabe precisar que, en el caso de los talleres con niños, los voluntarios de la Facultad de Educación realizaron los talleres de vínculo en los años 2018 y 2019. En el primer año, se realizaron únicamente los talleres de juego, recreación, música, baile y actividades gráfico-plásticas; mientras que, en el 2019 además de lo ofrecido en el año anterior, se incorporó el taller de mediación de lectura donde se realizaran lecturas compartidas con los niños. La experiencia que vamos a presentar data de este segundo año de voluntariado RSU y se centra únicamente en los talleres de mediación de lectura.

\section{La participación de los estudiantes en actividades de responsabilidad social universitaria}

Para comenzar, se brindará una definición de responsabilidad social universitaria (a partir de ahora RSU) que nos permita comprender el marco formativo de esta experiencia. Según la Ley Universitaria No30220 (MINEDU, 2014, p.54), la RSU “es 
la gestión ética y eficaz del impacto generado por la universidad en la sociedad debido al ejercicio de sus funciones: académica, de investigación y de servicios de extensión y participación en el desarrollo nacional en sus diferentes niveles y dimensiones". Asimismo, se ocupa de observar y atender la gestión de los impactos de la universidad en su contacto con el exterior e incluye los ejes de formación, investigación, extensión universitaria y administración (Vallaeys, 2014). Así, la RSU responde a un compromiso de la universidad con la sociedad, de interaprendizaje compartido, con principio ético y democrático, jugando un rol político innegable. Autores como Raza y Loachamín (2015) explican que la RSU pretende generar un trabajo compartido entre la universidad y la sociedad que genere un compromiso mutuo.

Los impactos de la RSU en la formación académica son variables, ya que pueden darse en los niveles de corto, mediano y largo plazo. Además, el reconocimiento de las demandas sociales que la universidad tiene con la sociedad, guía sus acciones y da respuestas pertinentes a estas demandas (García-Pliego y Olvera-García, 2011). Sobre la amplitud de alcance de la RSU, Naval y Ruíz-Corbella (2012) sostienen que la relación universidad - sociedad debe penetrar los ámbitos nacional y no restringirse únicamente a los ámbitos locales. Finalmente, para Valleys (2014, p. 110), la RSU es un esfuerzo por lograr la búsqueda de una "autonomía social compartida".

El enfoque de la RSU que tuvo la universidad donde se realizó la experiencia, en la época en que esta tuvo lugar, se basaba en un enfoque comunitario que buscaba "fortalecer el rol social y político de la universidad con el fin de contribuir a la gobernabilidad y a la sostenibilidad democrática, al reconocimiento positivo de nuestra universidad, a la disminución de toda forma de inequidad e injusticia y al cuidado del medio ambiente" (Sagástegui, Stonij, Velásquez, 2018, p. 1). En este sentido, la RSU facilitaba el ejercicio de una ciudadanía democrática y ética. Se promovía los aprendizajes "doble vía", que apelaba a una co-laboración y una coresponsabilidad, donde la universidad y la comunidad con la que se tenía vínculo, aprendían y se retroalimentaban mutuamente, a partir de una relación de igualdad. De manera general, la RSU puede permitir que en la universidad se reajusten mallas curriculares, metodologías de enseñanza e investigación (DARS, 2018).

El modelo educativo promovido desde la universidad donde se realizó está experiencia de RSU es por competencias. La RSU es reconocida y valorada como un eje transversal indispensable en la formación de los estudiantes de los niveles de pregrado y posgrado, que complementan los aprendizajes propuestos en los planes de estudios (PUCP, 2016). Sagástegui (2014) explica que la competencia académico profesional RSU tiene la finalidad de conjugar tres tipos de saberes que 
integran conocimientos conceptuales, procedimentales y actitudinales. En lo que respecta al saber conceptual este puede ser alcanzado cuando el estudiante logra conocer la realidad y aplicar el conocimiento adquirido. El saber procedimental hace referencia a las estrategias que el estudiante emplea para vincularse con las persona que interactúa de forma flexible, poniendo en práctica los saberes conceptuales adquiridos y tomando decisiones para la mejora continua, dando solución a problemas. Finalmente, el saber actitudinal está relacionado a la conjunción de los saberes académicos y profesionales, que permite que el estudiante se sensibilice ante una realidad, reaccione y proponga actuar frente a los problemas sociales. Las actividades de RSU son una oportunidad para que los estudiantes se relacionen de manera interdisciplinaria, trabajen de manera colaborativa, tomen decisiones para dar solución a problemas integrando los saberes interdisciplinarios, valorando los saberes que son generados por las personas con las que interactúan.

Ahora bien, en este punto se considera fundamental precisar el enfoque formativo que tuvieron los estudiantes voluntarios de esta experiencia. La Facultad de Educación tiene un enfoque crítico reflexivo basado en la pedagogía crítica. Uno de sus propósitos es formar docentes que conozcan la realidad educativa peruana en sus diferentes contextos y que tengan experiencias de RSU que les permitan conocer y enfrentar problemas educativos que afectan a la infancia y a la adolescencia, así como a los diferentes actores educativos ${ }^{2}$. En suma, se puede concluir que la RSU abre posibilidades para que el estudiante conozca e interactúe con diversos actores y realidades, contribuyendo al desarrollo integral del estudiante, que alcanza los niveles personal, académico y profesional.

\section{Los talleres de lectura con niños de la comunidad de Sacsamarca}

¿Por qué leemos a los niños? ¿Por qué gastamos una enorme energía humana y económica alrededor del acto de leerles? Nosotros no leemos los textos a los niños para que se conviertan en buenos lectores, sino porque sabemos que esas lecturas les permiten ubicar algo fundamental para ellos: el descubrimiento de que los textos son cosas que tienen un sentido, cantidad de sentidos y que cada sujeto debe trabajar un poco para llegar a construir el sentido en su espíritu (Cabrejo, 2001, p.15).

2 La información complementaria sobre las carreras que brinda la Facultad de Educación y sus propósitos pueden encontrarse en: http://facultad.pucp.edu.pe/educacion/ 
Se inicia este apartado evocando la cita de Evelio Cabrejo sobre el propósito de la lectura compartida con niños porque nos ayuda a fundamentar lo que se describirá a continuación: una experiencia de la implementación de un taller de mediación de lectura dirigido a niños de la comunidad de Sacsamarca con el objetivo de que vivan la experiencia del acercamiento a la lectura y el encuentro con el libro a través del otro, lo que les permitió compartir múltiples lecturas compartidas y conversaciones en torno a esas lecturas. Este taller tuvo la duración de 6 meses, dividido en 12 sesiones, que se extendieron entre los meses de junio a diciembre del 2019. Para poder comprender cómo se genera este taller es necesario contextualizarlo en su historia.

Durante los años 2018 y 2019, la DARS y la Facultad de Educación unieron esfuerzos para constituir un equipo de estudiantes voluntarios que integraran los talleres permanentes de la iniciativa "Como Jugando", que era una actividad que estudiantes de otras facultades habían desarrollado desde el año 2015 con niños de la comunidad de Sacsamarca. Este vínculo contribuyó a que estudiantes y una docente de la Facultad de Educación se organizaran para participar del voluntariado, lo que contribuiría a la sostenibilidad de los talleres infantiles, pero enfocándose en una óptica propiamente educativa. Cabe destacar que hasta el año 2017, los talleres que se realizaban con los niños de la comunidad se diseñaban con una metodología basada en el arte y el juego, donde el grupo de voluntarios estaba conformado con estudiantes que provenían de diversas carreras. En el año 2018, como resultado de la convocatoria de la DARS en vínculo con la Facultad de Educación, el grupo de voluntarios se reconfiguró y estuvo integrado por estudiantes de las especialidades de educación inicial y primaria, sin descuidar el trabajo interdisciplinario que era garantizado con el acompañamiento de la DARS.

Los talleres de vínculo se realizaban en la Plaza de Armas del centro poblado, seis o siete veces al año, entre los meses de mayo y noviembre que era la temporada de no lluvias. Durante esta experiencia, los talleres se ejecutaban dos días durante cada visita, desarrollándose los días viernes y sábados con una duración de 2 horas con 30 minutos aproximadamente. Las actividades se iniciaban con un llamado al agrupamiento de los niños a modo de convocatoria y marcaba el inicio de las actividades, luego de iniciada la concentración se daba paso a las actividades como rondas y juegos al aire libre que se empleaban como descarga, y a continuación, se realizaba la propuesta de estaciones con diversas actividades que incluían el baile, el canto, las gráfico plástica, movimiento y deporte, actividades literarias, entre otras. Las edades de los niños oscilaban entre los 3 a 12 años, pero en el caso de los más pequeños (los menores de 6 años) iban casi siempre en compañía de 
sus hermanos mayores o madres. Cada visita tenía un objetivo que era planteado por los voluntarios durante la planificación de los talleres; por ejemplo, en la visita 2 el objetivo estuvo centrado en "fomentar prácticas de respeto mutuo entre los niños de Sacsamarca para generar una convivencia armónica y vínculos afectivos" (Planificación junio). En el caso del taller de mediación tuvo sus propios objetivos vinculados a la lectura:

- Generar un espacio de lecturas compartidas para brindar el acceso al libro en la comunidad de Sacsamarca.

- Emplear la herramienta mediación de lectura para ejecutar los talleres de lectura.

- Implementar un sistema de préstamos de libros a domicilio para promover la circulación de los libros.

La metodología de los talleres se basó en una propuesta de 4 estaciones con actividades donde los niños tenían la oportunidad de elegir de manera libre y de acuerdo a sus intereses, que les permitiera disfrutar de la actividad de su preferencia, eligiendo una de las estaciones y podían circular de manera libre entre una y otra estación a lo largo de la jornada; por ejemplo, podían permanecer media hora en el taller de juego para luego dirigirse al taller de gráfico plástica, lectura o movimiento. En el caso del taller de mediación de lectura se destaca que hacía parte de esta propuesta y era muy demandado por los niños. Las actividades del día culminaban con un compartir donde participaban los niños y los voluntarios.

La propuesta del taller de mediación de lectura permitió que los niños tuvieran acceso a los libros y conocieran otros títulos que hasta el momento ellos no habían podido leer. De manera general, este taller permitió democratizar el acceso de los niños a la lectura, sobre todo porque se trataba de libros a los que tenían acceso fuera del ámbito de la escuela, que hasta ese momento era el único en el que tenían contacto con los libros y la lectura. Cuando se conversó con las familias, ellos manifestaron que no realizaban lecturas con los niños salvo las que les solicitaban en la escuela, pero si tenían prácticas de narración oral con historias, leyendas o cuentos. Cabe destacar que desde que se inició el voluntariado en el año 2018, los niños solicitaban que se realizaran lectura al inicio y al final de los talleres, demostraron interés por participar de lecturas compartidas en voz alta y de los momentos consagrados a la lectura. Esta demanda de los niños fue el motivo principal que impulsó la planificación de un taller de lectura para el año 2019. En colaboración con los voluntarios se realizaron las siguientes actividades (Informe del Resumen ejecutivo de las ponencias de las mesas temáticas ENARSU, 2019): 
- Lecturas en voz alta realizadas de manera compartida con niños de diferentes edades

- La mediación de lectura fue realizada fundamentando cada una de las acciones en experiencias o publicaciones académicas de autores reconocidos en el tema.

- En algunas oportunidades se realizaron narraciones orales a demanda de los mismos niños o ellos compartieron las narraciones que conocían de su comunidad. Esta actividad se realizó como un complemento a las lecturas que se ofrecían.

- Préstamo de libros a domicilio que permitió la circulación de los textos en bolsas viajeras.

Con la finalidad de impulsar el taller, se partió de la idea que la mediación de lectura es un proceso intencionado de promoción de la lectura con actividades programadas y en base a la conformación de un itinerario de lectura que los niños construían de manera autónoma, que tiene como finalidad promover la práctica sociocultural de la lectura (Sandoval y Landaverry, 2019). Asimismo, las actividades de lectura fueron planificadas y ejecutadas a largo plazo, con la participación activa de los niños, teniendo en cuenta los modos de realización de la lectura, así como en las interacciones que son resultado de ella (Munita, 2014). Asimismo, se sustentó la necesidad de concebir la lectura como una práctica social del lenguaje, que se concibe y se construye en situaciones reales de comunicación, pero que además, es contextualizada y tiene propósitos comunicativos que la guían (Rodríguez, 2014). En cuanto a los espacios donde se desarrolla la práctica lectora con niños se comprendió que era necesario ampliar la experiencia de los niños a espacios no escolares porque contribuye a consolidar su interés por la lectura (Aliagas, 2009).

Se tuvo en cuenta la importancia de la exposición de los niños al contacto con el libro de manera temprana y la familiaridad con las lecturas que se da a través de la experiencia de la lectura compartida y que se realiza para ellos en voz alta con el acompañamiento de un lector más experimentado, en diversos contextos como son los familiares y escolares, así como la experiencia con el acceso a las bibliotecas (Reyes, 2008). En el caso de los niños de la comunidad de Sacsamarca, es importante tener en cuenta, que ellos solo tenían contacto con el libro a través de la escuela y nunca habían acudido a una biblioteca, por lo que el taller representaba para ellos otra forma de leer y acercarse a los libros. Es necesario precisar que el aseguramiento de las prácticas de lectura en los niños se da a través de un 
proceso paulatino y progresivo, en el marco de acciones de fomento de la lectura, donde la mediación tiene un rol fundamental (Marlasca, 2015).

Finalmente, los voluntarios tenían conocimiento de la importancia de crear un vínculo entre los niños y la lectura a través de la voz, durante las lecturas en voz alta, que pueden ser un recurso potente para generar emoción durante las lecturas (Reyes, 2007). Cada palabra escuchada por el niño es como el puente que lo conecta hacia lo escrito, oralidad y lectura se complementan. Este aspecto es importante porque se hacía alusión a la "envoltura emocional" que se genera en la mediación de la lectura con niños pequeños porque se establecerá una relación entre lo escrito y lo afectivo, lo que permite que los niños asocien esas lecturas compartidas a vivencias positivas y rompan con la idea de que los libros son objetos lejanos e inaccesibles que a veces relacionan con elementos extraños o amenazantes (Bonilla, Goldin y Salaberría, 2009).

Como se precisó en párrafos anteriores, el taller de mediación de lectura se generó a demanda de los mismos niños y sus familias. Ya desde el año 2018, en el marco de los talleres de "Como Jugando", ellos comenzaron a escuchar las lecturas de las voces de los voluntarios. Este ejercicio permitió que los niños realizaran prácticas de lectura entre las que se puede mencionar la exploración libre de los libros, la elección de los mismos y las conversaciones que se generaban luego de culminadas las lecturas. Ya en el 2019, una vez instalado el taller, se procedió a la concepción de un sistema de préstamo de libros a domicilio adaptado a la realidad de la comunidad, que tuvo éxito y fue apreciado por los niños y sus familias, permitiendo el préstamo a través de bolsas viajeras de libros que fueron acogidas por las familias. Una de las alianzas estratégicas para lograr la realización del taller fue trabajar con la comunidad y los padres de familia. A nivel de la comunidad, los talleres fueron realizados en las instalaciones de la Municipalidad; mientras que, el acervo de libros con los que se contaba pudo guardarse en casa de una de las familias que habitaba cerca del lugar. A nivel de las familias hubo un apoyo en la circulación del préstamo de libros que garantizaba la lectura y el retorno, dado que los préstamos se realizaban con una duración de un mes y esto necesitaba que los libros pudieran permanecer con las familias durante ese tiempo y se requería un cuidado del material. Las familias también contribuyeron con la lectura de los libros porque comprendieron que podían realizar la lectura repetidas veces y que los hermanos mayores podían garantizar las lecturas en voz alta a los más pequeños.

Como en todo proyecto, esta iniciativa tuvo los siguientes resultados alcanzados (Informe del Resumen ejecutivo de las ponencias de las mesas temáticas ENARSU, 2019): 
- Los talleres permitieron compartir las lecturas a partir de los propios intereses de los niños.

- El funcionamiento del sistema de préstamo de libros en las bolsas viajeras, que se realizó mensualmente y que se registró a través de un listado simple que incluía el título de la obra y el nombre del niño.

Los talleres de mediación de lectura se concibieron teniendo como eje central al niño y se abrió la posibilidad de la exploración previa del texto como base de la mediación de lectura. Así, los niños se acercaban a la mesa y revisaban los libros, los iban leyendo de manera aleatoria para conocer su contenido, los compartían con sus compañeros y manifestaban sus intereses, para finalmente solicitar la lectura en voz alta al voluntario. Este proceso culminaba con una conversación abierta sobre las lecturas, vinculándolas a la experiencia que ellos vivían y deseaban compartir (Chambers, 2007). Este aspecto fue fundamental, dado que las lecturas compartidas permitieron que los niños se expresaran a su manera y con los recursos que tenían sobre sus emociones, sentimientos, ideas, preguntas o cuestiones que los interpelaba, así como vincularon las lecturas que se realizó en el taller con aquellas experiencias que ellos habían tenido en la escuela, por ejemplo, realizaban resúmenes de las obras y recomendaciones para que otros niños orientaran sus elecciones en función a la experiencias de otros niños.

Con respecto al sistema de préstamo de libros se pudo llevar a cabo gracias a la donación de estudiantes y docentes de la universidad. En total se conformó un acervo de 80 libros que incluían libros ilustrados infantiles, libros álbum, cuentos, libros informativos, algunos libros juego y enciclopedias. La selección de las donaciones y el etiquetado estuvo a cargo de los voluntarios, quienes prepararon los libros en Lima y los condujeron en una maleta roja hasta Sacsamarca. Esta maleta era reconocida por los niños y se generó un ritual en los talleres de mediación, dado que eran los mismos niños los que esperaban la llegada de la maleta y su apertura, luego de la exploración y los primeros comentarios sobre los autores o las temáticas se comenzaba a leer en voz alta, para finalmente conversar sobre lo leído. El diseño y ejecución del sistema de préstamos consideró el contexto sociocultural de los niños, así como sus prácticas de lectura familiar y escolar. Se conversó con los niños con respecto a la importancia de la circulación de los libros, que era un bien compartido y que beneficiaría a todos, de manera que su retorno era necesario.

La experiencia que se evoca en este artículo no estuvo exenta de factores que facilitaron y dificultaron el proceso (Informe del Resumen ejecutivo de las ponencias de las mesas temáticas ENARSU, 2019). Entre los factores que facilitaron los 
talleres se puede mencionar la preparación en didáctica de la lectura que tenían los voluntarios de la Facultad de Educación, de ambas especialidades, así como la colaboración de las familias para hacer funcionar el sistema de préstamos. En cuanto a la planificación de los talleres fue fundamental el trabajo en equipo que se realizó de manera coordinada, asegurando que todos los voluntarios tuvieran la oportunidad de planificar, ejecutar y evaluar el taller. Otro aspecto importante fue la consolidación progresiva del préstamo de libros a la familias, dado que fue progresivo y se fue incrementando la cantidad de libros a prestar a medida que se aseguraba el retorno de los anteriores. Finalmente, el apoyo de los acompañantes de la DARS y la asesora permitió que los estudiantes voluntarios pudieran proponer estrategias creativas en las lecturas y reflexionaran sobre lo vivido en las reuniones de reflexión que se realizaban una vez culminadas las sesiones. Entre los factores que dificultaron los talleres se puede mencionar el pequeño acervo de libros que se tenía, dado que limitaba la variedad de los mismos y el no contar con una persona de la comunidad que pudiera continuar con los préstamos a domicilio durante el periodo en que los voluntarios no estaban presentes en la comunidad. Para continuar con una experiencia similar sería conveniente tener una biblioteca municipal donde se puedan albergar los libros y el taller.

\section{Los aprendizajes resultados de las experiencias de RSU}

Como se indicó en la introducción de este artículo, con la finalidad de sistematizar la experiencia de docentes y estudiantes se recurrió a la consulta de las programaciones de talleres y se realizó una entrevista grupal con algunos de los estudiantes voluntarios en agosto del año 2020. Las preguntas se organizaron en dos partes: primero se preguntó sobre lo que significó el taller de mediación de lectura con los niños y las interacciones resultado de las lecturas compartidas y las conversaciones; y posteriormente, se abordó lo concerniente al sistema de préstamos de los libros.

Ante la pregunta sobre los recuerdos que tenían con respecto al inicio del taller, los testimonios de los estudiantes ilustraron el entusiasmo y el compromiso de poder implementarlo en respuesta de las demandas y necesidades de los niños:

Recuerdo mi entusiasmo y emoción por participar del taller porque era la primera vez que participaba en un voluntariado en provincia [...], me sentía tensionada porque quería dar lo mejor de mí y no sabía lo que podía pasar(E1). 
Para mí ha sido una experiencia bastante enriquecedora y nueva, fue la primera que íbamos un taller de lectura y ellos siempre se emocionaban cuando oían eso, nos buscaban para leerles y mirar los textos. A veces sentía inquietud porque me preocupaba el tema de mi voz, cómo voy a leerles, cómo tenerlos tan atentos; entonces, con mis compañeros que compartimos el taller íbamos viendo (E2).

En mi caso ha sido una experiencia gratificante y muy bonita porque pude ver ciertas cosas que llevamos a la práctica, pude ver ciertas cosas que vimos en el curso de Comunicación sobre los libros y la biblioteca. Cuando fuimos implementando el taller de mediación de lectura realmente implementamos esos espacios de lectura (E3).

Para mí fue mi primera vez en un voluntariado en provincias, en Ayacucho, fue emocionante porque era otro contexto y otra cultura. No solamente vas a enseñar, sino sobre todo a aprender de ellos. Cuando iniciamos el taller de lectura vi cómo los niños se animaban a ir [...]. Uno de los recuerdos que tengo es cuando al final de los talleres vi a un niñito como le enseñaba a leer a otro niñito. Era gratificante, no solo te quedas con un aprendizaje propio, sino también colectivo. Era increíble cuando llevaban los libros a sus casas y sé que tuvimos algunas dificultades, pero lo importante era ver cómo veían a los libros y los leían (E4).

La propuesta de empezar a prestar libros, hacer el maletín y el inventariado tuvo sus inicios antes porque los anteriores voluntarios leían a los chicos. Recuerdo que el primer día que llegamos a Sacsamarca y leímos un cuento en la canchita y vimos su interés en su cara, que a la vez les gustaba y no era algo que ocurría todos los días en su contexto. De allí creo que se fue formando ese interés de los pequeños, creo que en el 2019 era imposible no responder a eso, no hacer algo al respecto, para continuar este hilo de lectura que se había formado anteriormente (E5).

Luego, se les preguntó sobre sus percepciones de la acogida que los niños tuvieron por el taller de lectura. Los estudiantes mencionaron que:

Los niños nos esperaban, querían que se realizará el taller, con las lecturas pudimos conquistar una parte del corazón de cada pequeño (E1).

Cuando llegamos a Sacsamarca, los niños nos esperaban y nos preguntaban ¿qué van a leernos?, eso era parte de las interacciones que teníamos con ellos. Recuerdo el asombro de los niños durante las lecturas, tengo fotos donde ellos están sorprendidos o callaban a los otros niños para encontrar el silencio en las lecturas. Era raro ver eso, en el momento del cuento había ganas de los niños de escuchar [...] La gran mayoría tenía una buena recepción de este taller (E5). 
Era muy gratificante ver a los niños, yo creo que lo han tomado de la mejor manera. Hemos abordado problemáticas del contexto, era diversificado para que ellos se sientan acogidos y parte del proyecto, no como imponiéndose. Mediante la lectura íbamos tratando de convivir, que ellos se dieran cuenta que estábamos juntos leyendo (E4).

Creo que poco a poco a los niños los hemos ido cautivando con el taller de mediación de lectura porque en cada viaje que hacíamos veíamos cómo los niños nos pedían más lecturas. Nosotros habíamos puesto unos límites para los préstamos, empezamos prestando poquito y llegando a la mitad del tiempo ya eran más los que tenían. Íbamos estableciendo más criterios para evaluar cómo prestar más libros (E3).

Otro aspecto a destacar es la evolución que tenían los niños como lectores:

Ellos empezaron a darse cuenta que tenían que cuidar los libros porque era para prestar a otros, poco a poco fueron interiorizando esas cosas (E1).

Con respecto a la implementación de los talleres, los estudiantes se agruparon en parejas y fueron planificando los talleres, así eligieron diversas lecturas para proponer a los niños:

Recuerdo que cuando hicimos una actividad con E4, antes del viaje nos designamos tareas para realizar la lectura, pero el día que llegamos a Sacsamarca empezamos a mencionar acuerdos previos a la lectura y empezamos a recrear la historia que ya habíamos leído (Atrapados, Oliver Jeffers, Fondo de Cultura Económica). Quisimos dar algunas recomendaciones al cuento, sobre todo la escucha activa, de allí empezamos a leer. Ese día estuvimos en el auditorio y había bastante bulla, fue un poco difícil esa vez (E5).

Uno de los retos fue, a pesar de haberlo trabajado en la universidad, eso de trabajar en equipo fue una experiencia distinta en el voluntariado. Cuando queríamos dar muchas ideas nos costaba ponernos de acuerdo, incluimos las ideas de ambas, pero luego veíamos que podíamos haber modificado algunas cosas de la propuesta. Eso fue un reto, sobre todo la preparación para llevar el taller, plantearnos bien en claro el objetivo, darles la experiencia de la lectura, de despertar su interés (E1).

Además de los retos, los estudiantes evocaron la importancia de la toma de decisiones durante el taller para la solución de problemas:

Una vez realizamos la lectura y nos sobró tiempo, mi pareja hizo una actividad de baile porque no sabíamos qué más hacer con los niños. Para programar la lectura 
fue emocionante, pero cómo es en el proceso de la lectura con los mismos niños las cosas podían variar, a veces teníamos que contener un poco sus emociones cuando conversábamos. Eso era muy difícil, tener que tomar decisiones para enfrentar (E4).

Yo visualicé dos retos para la preparación de los talleres: en primer lugar fue especificar los acuerdos de convivencia para la lectura porque los necesitábamos para ejecutar los talleres. Y otro de los retos fue plantear lecturas que se ajuste a los intereses de todos porque teníamos niños de diferentes edades, con diferentes gustos y necesidades (E3).

Con respecto a la realización de las lecturas en voz alta, los estudiantes mencionaron que a veces sucedían imprevistos durante las interacciones en la lectura:

Algunos niños interrumpían porque querían saber qué pasaba, en el momento del espacio de la lectura preguntaban, ¿qué pasa luego?, ellos querían saber lo que iba a pasar con el personaje y seguían la lectura. Inclusive, cuando terminábamos de leer nos pedían el texto para revisarlo, tenían atención pero interrumpían bastante durante la lectura. Era como una demanda para ver los textos y elegir cuál podrían llevarse a casa (E2). Justo cuando hicimos la lectura ellos estaban esperando para dibujar, se emocionaban, se imaginaban, era divertido. Recuerdo que en el taller de lectura ellos querían agarrar todos los libros cuando abríamos la maleta, ellos nos preguntaban bastante si íbamos a volver. A mi gustó que mientras revisaban el libro me pedían que les lea una parte que les interesaba (E3). Era lindo ver sus caritas de emoción e intriga, como mencionaron mis compañeros. Era gratificante ver cómo a través de las lecturas que les leíamos ellos nos contaban sus experiencias, nos decían sus dudas, preguntaban (E1).

Sobre las preferencias de los niños por las lecturas, los estudiantes mencionaron:

Se notaba que los niños no conocían los libros, se asombraban y pedían, ellos querían que nosotros les leyéramos el cuento. No los escuchaba leer solitos, los veía que hacían predicciones con las imágenes, ellos sentían un gusto cuando les leíamos. Creo que ellos querían escucharnos, veían nuestras manos, escuchaban las voces que hacíamos, siento que los niños necesitaban hacerse un mundo en su mente para imaginarse las lecturas [...]. Siempre que llegábamos nos preguntaban qué íbamos a leer y siempre estaban esperando saber quién iba a leer [...]. Recuerdo que la escuelita no tenía biblioteca y si había libros no los prestaban, entonces darles nosotros los libros fue muy bueno para ellos (E5).

Los niños exigían que nosotros les leyéramos un cuento, siento que les gustaba el momento de pedirnos la lectura, era un momento agradable porque siempre en 
el taller hemos tratado de crear un espacio seguro para interactuar. El momento de la lectura era un momento esperado por los niños (E6).

Entre las demandas de los niños estaba el préstamo de libros:

Ellos pedían más libros para prestar, pero no teníamos tanto y era más complicado para asegurar la devolución (E1).

Yo creo que fue una gran propuesta el préstamo de libros porque íbamos a permitir que se acerquen más a la lectura, que ellos mismos escojan sus libros y se los lleven. Para los niños fue muy enriquecedor, pero para nosotros significó un gran trabajo porque no solo era registrar los libros, sino que había que llevar una metodología para el préstamo, hacer el seguimiento para la devolución, el seguimiento. Al iniciar el taller teníamos que recordar que los trajeran de vuelta, recién al final como que ya habían entendido que tenían que volver (E6).

Fue muy gratificante el préstamo de libros y una labor muy ardua, en algunos casos fue complicado porque préstamos los libros a niños que ya no volvieron al taller y tuvimos que hacer seguimiento, pero al final lograron consolidar la idea del préstamos y aprendieron del proceso (E4).

En relación a los esfuerzos como equipo hemos tenido que hacer más cosas, tuvimos que separar un tiempo durante la semana para asistir a las reuniones de planificación. Recuerdo que habíamos pensado cómo hacer el préstamo, pensamos en hacer un carnet para que ellos fueran lectores, teníamos que ver el registro de los préstamos y no sabíamos si usar cuaderno, un cartel donde los niños pudieran marcar los préstamos y ellos mismos vayan viendo lo que iban leyendo y devolviendo [...]. Cuando nos dieron los libros fue muy complejo seleccionar los libros y etiquetarlos porque debíamos darle un orden a los libros de la maleta. Trasladar la maleta también fue complicado porque cada vez iba llenándose más. Nos reuníamos para ver quién prestaba y quién recibía los préstamos, y justo coincidía con la entrega del refrigerio, se hacía un ajetreo total, pero creo que al final ese trabajo extra fue bien recompensado cuando veíamos a los niños leer (E5).

En cuanto al desempeño docente en el marco de la RSU, los estudiantes destacaron que ser flexible era una característica necesaria en el trabajo con niños y que apoyaba la apertura para dar respuesta a las demandas de esta población. El diálogo y la comunicación son aspectos que también valoraron para promover una convivencia armónica con la comunidad. Todos estos aspectos aportaron a su desempeño docente, asociado a la capacidad de ser resilientes y aprender en diversas situaciones, inclusive del error: 
Las complicaciones son generadoras de lo que se conoce como ensayo y error, estas son inevitables cuando se introduce en el mundo de la educación, y debería ser parte de nuestras vidas para ser conscientes de que siempre debemos tener esa capacidad de resiliencia en potencia (E5).

En lo que respecta a su propia práctica y a la mejora continua de su ser docente los estudiantes manifestaron que es importante:"Tener más confianza al momento de dirigir las actividades" (E4).

Otro aspecto que consideraron fundamental fue la planificación de actividades y su capacidad de ser flexibles para adaptarse a las diversas situaciones y personas, colaborar con sus compañeros e integrar las demandas de los niños:

Aprendí que debo ser flexible, incluso más de lo que pensé, pues si las cosas no están resultando, y estamos en la posibilidad, cambiarlo, hacer arreglos y no frustrarse con eso (E1).

Destacaron también la necesidad del diálogo intercultural y la comunicación asertiva con los niños, asimismo tener conocimientos del quechua fortaleció la comunicación con los niños, el acercamiento, la escucha activa y la atención de situaciones imprevistas:

Saber quechua me favoreció para vincularme con ellos, por ejemplo, surgían canciones conocidas y las cantábamos (E5).

También evocaron que la oportunidad de recibir un acompañamiento formativo del equipo de la DARS porque los retroalimentaron de manera permanente sobre su accionar y les permitió trabajar de manera interdisciplinaria, así como mirar sus propios desempeños reflexionando sobre su actuar:

De esta experiencia yo aprendí a trabajar en equipo, no trabajaba por un objetivo propio no hubiésemos logrado nada. Concluyó que tener un soporte en el grupo es que si compartes tu idea puedes ser considerado, todos van a trabajar para sacar el taller adelante, si alguien necesita ayuda vamos a apoyar (...) Sobre el taller de mediación de lectura me llevo el recuerdo de estar bien emocionalmente para poder conectar con ellos y leerles, influye en la manera en cómo nos relacionamos con los niños, es importante estar seguro que lo que estamos haciendo les sirve a ellos, los apoyamos a desarrollar sus habilidades de lectura (E5). 
Otro aspecto que mencionaron fue el de los aprendizajes propios para la toma de decisión en procesos complejos:

Yo he aprendido que todo reto, por más complicado que pueda verse, si lo pensamos bien y actuamos va ser beneficioso para los niños (...): El préstamos de los libros fue un gran reto, la logística fue complicada, pero lo hicimos a pesar de que nosotros estábamos estudiando y lo logramos porque los niños se interesaban más por las lecturas, se juntaran y compartían entre ellos, cuando les dejábamos los libros nos contaban que se juntaban a leer o se prestaban el libro, se intercambiaban, todo esto era de gran beneficio para ellos (E1).

Lo que me llevo es el trabajo en grupo de manera coordinada, no perder la calma si pasa algo, la necesidad de contactarnos con la comunidad porque los necesitamos, nos ayudan cuando les pedimos. Nosotros hemos aprendido de la implementación del taller y de los préstamos, queríamos que los niños repliquen la lectura en su comunidad, que sientan que la lectura puede verse desde varios ámbitos, la lectura está en los libros y en los que les rodea, ellos ven la lectura en cada rincón de su casa y de su comunidad (E4).

Para finalizar, las experiencias que los estudiantes nos han brindado ilustran la importancia del acompañamiento formativo de la DARS, el rol activo de los estudiantes en este proceso, así como la posibilidad de que los cursos sean generadores de conocimientos y experiencias para aplicar en el voluntariado y la importancia de la consolidación de los equipos y el trabajo en grupo, la convivencia, la apertura y el diálogo, que son aspectos que fueron aspectos necesarios para asegurar la sostenibilidad de los talleres en esa experiencia.

\section{Conclusiones}

De manera general, los voluntarios del taller de mediación de lectura en el marco de las actividades de "Como Jugando" han aprendido a generar espacios de lectura compartida y acompañaron los procesos de apropiación de la lectura de los niños de la comunidad, respetando su contexto sociocultural y sus procesos lectores. Asimismo, se observó que trabajaron en alianza con las familias, de manera que los préstamos de libros pudieran ser ejecutados. En un contexto de limitado acceso a los libros y la lectura, este taller permitió que se compartieran experiencias de lectura con los niños, desde el inicio fueron ellos los que las solicitaban. La planificación 
de los talleres consideró el contexto, las personas y sus necesidades, así como los intereses que ellos manifestaban.

Los talleres de mediación de lectura contribuyeron a que los niños se acercaran a los libros, los explorarán, los leyeran, los compartieran y expresaran sus sentimientos, así como sus experiencias lectoras. En un inicio la lectura era asociada a experiencias escolares y luego fueron conversando de manera más libre sobre lo leído. Se mantuvo una relación de cuidado entre los voluntarios y niños de la comunidad, lo que facilitó la generación de espacios de conversación e interacción positiva en torno de las lecturas. El juego fue considerado como elemento integrador para la buena convivencia y mantenimiento del taller de mediación de lectura, que fue una herramienta que permitió generar los espacios de lectura compartida y, gracias a este acompañamiento, los niños pudieron construir sus prácticas lectoras de manera progresiva y autónoma. Los procesos reflexivos se daban desde la elección de los libros hasta la restitución de las experiencias vividas a raíz de las lecturas.

El diseño y ejecución del sistema de préstamos consideró las prácticas y rutinas de los niños y sus familias, además se conversó sobre la importancia de la circulación de los libros porque eran de todos. El sentido de compartir y de servicio arraigado en la comunidad fue la base para el éxito del sistema de préstamos: reciprocidad, intercambio, agradecimiento, sentido de pertenencia, apropiación de las lecturas. Otro logro importante es que los niños se formaron como lectores, eligieron sus libros y comentaron sobre ellos, además compartieron sus propias experiencias. El sistema de préstamos impulsó esta práctica.

Los estudiantes declaran que los aprendizajes resultado de la experiencia de RSU se han dado a varios niveles, tanto el personal como el profesional. Personalmente, se autoperciben más empoderados y empáticos, lo que les permite comunicarse con las personas con las que interactúan y tomar decisiones frente a problemas que se presentan; en tanto, a nivel profesional, destacan la importancia de desarrollar de manera permanente su ser docente gracias a los intercambios con sus compañeros, los aprendizajes colaborativos con los niños, los interaprendizajes logrados entre los voluntarios, el asesoramiento de la docente y el acompañamiento formativo de la DARS. Los estudiantes han desarrollado diversas competencias entre las que se puede destacar: el diseño de sesiones para el trabajo con niños en escenarios educativos no formales, una diversidad de formas de planificación y su diversificación dependiendo de las personas y sus necesidades, flexibilidad y creatividad en sus propuestas educativas integrando el juego, el arte y la lectura, así como tomar decisiones y brindar respuestas a problemas que se presenten o situaciones imprevistas. Asimismo, otro aspecto importante es la comunicación eficaz que han 
logrado establecer con los niños y sus familias, promoviendo un diálogo abierto y democrático que es parte fundamental para sacar adelante el vínculo colaborativo, integrando los propósitos de la RSU en su formación y, sobre todo, se han sido empático al valorar los aprendizajes que les ha brindado la población.

Finalmente, esta experiencia muestra que la RSU es fundamental en la formación de los estudiantes universitarios, sobre todo en el caso de la formación inicial docente. Asimismo, la RSU aporta al currículo crítico y reflexivo que promueve la Facultad de Educación porque permite que los estudiantes tengan experiencias educativas en diferentes escenarios de aprendizaje, con poblaciones diversas y que den respuesta a problemas educativos variados. Los estudiantes voluntarios cuestionan los conocimientos aprendidos, los aplican y fortalecen en su confrontación con la realidad, en la toma de decisiones y en la escucha a los actores educativos. El trabajar de manera coordinada y colaborativa con la DARS ha permitido que se alcance la interdisciplinariedad en el trabajo educativo, así como fortalecer la dinámica teoría-práctica que es fundamental en la formación inicial de los docentes de educación básica. Por último, se considera fundamental destacar que el vínculo interunidades y el trabajo colaborativo entre docentes-estudiantes han contribuido a fortalecer esta experiencia. 


\section{Referencias bibliográficas}

Aliagas, C. (2009). Aprendiendo a leer y a escribir con la canguro. En D. Cassany (ed.), Para ser letrados. Voces y miradas sobre la lectura (pp. 165-177). Barcelona: Paidós Educador.

Bonilla, E., Goldin, D. y Salaberría, R. (2009). Bibliotecas y escuelas. Retos y posibilidades en la sociedad del conocimiento. España: Océano.

Chambers, A. (2007). DIME. Los niños, la lectura y la conversación. México: Fondo de Cultura Económica.

Del Castillo, C. (2018). Aprendiendo en el encuentro, allin kawsanapaq. Punto Edu (450), pp.15. Recuperado de: file://D:/Users/sandoval.cm/Downloads/388806510PuntoEdu-Ano-14-numero-450-2018.pdf

Dirección Académica de Responsabilidad Social (2019). Informe del Resumen Ejecutivo V ENARSU. Documento de trabajo. Lima: PUCP.

Dirección Académica de Responsabilidad Social (01 de febrero del 2018). ¿Qué es la DARS? Recuperado de: http://dars.pucp.edu.pe/quienes-somos/que-es-la-dars/

Dirección Académica de Responsabilidad Social (01 de febrero del 2018). Reconstruyéndonos. Recuperado de: http://dars.pucp.edu.pe/proyectos/reconstruyendonos/ objetivos-y-metodologia/

Dirección Académica de Responsabilidad Social (2018). Protocolo de vinculación con cursos dirigido a docentes en el marco del Convenio Sacsamarca. Documento de trabajo interno. Lima: PUCP.

Dirección Académica de Responsabilidad Social (2018). Planificación de las actividades e informes de los estudiantes. Como Jugando (mayo, junio, julio). Documento de trabajo interno. Lima: PUCP.

Espinoza, J., Escudero, A., Mamani, A., y otros (2018). Historia de la comunidad campesina de Sacsamarca. Diálogo, memoria y reconocimiento. Lima: Fondo Editorial de la Pontificia Universidad Católica del Perú.

Gasca-Pliego, E. \& Olvera-García, J.C. (2011). Construir ciudadanía desde las universidades, responsabilidad social universitaria y desafíos ante el siglo XXI. Convergencia, Revista de Ciencias Sociales, 56, 37-58. Recuperado de http://www.scielo.org.mx/ scielo.php?script=sci_arttext\&pid=S1405-14352011000200002

Marlasca, B. (2015). El papel de la biblioteca pública en la formación de lectores. En S. Sánchez-García, S. Yuvero (eds.), Las bibliotecas en la formación del hábito lector (pp. 91-126). Cuenca: Universidad de Castilla-La Mancha.

Ministerio de Educación (2014). Ley Universitaria No30220. Lima: MINEDU. 
Munita, F. (2014). El mediador escolar de lectura literaria. Un estudio del espacio de encuentro entre prácticas didácticas, sistema de creencias y trayectorias personales de lectura (Tesis para optar el grado de Doctor). Universidad Autónoma de Barcelona, España.

Naval.C. \& Ruiz-Corbella, M. (2012). Aproximación a la responsabilidad social universitaria: la respuesta de la universidad a la sociedad. Bordón, 64 (3), 103-115. Recuperado de https://dialnet.unirioja.es/descarga/articulo/4016119.pdf

Petit, M. (2016). Leer el mundo: Experiencias actuales de transmisión cultural. México: Fondo de Cultura Económica. Petit, M. (2001). Lecturas: del espacio íntimo al espacio público. México: Fondo de Cultura Económica.

Pontificia Universidad Católica del Perú (2016). Modelo Educativo PUCP. Lima: PUCP.

Raza, N.A. \& Loachamín, J.J.M. (2015). La responsabilidad social universitaria: Componente fundamental en la formación de los futuros docentes de la carrera de Pedagogía, de la Universidad Politécnica Salesiana. Alteridad. Revista de Educación, 10 (1), 8699. doi: 10.17163/ait.v10n1.2015.06

Rodríguez, S. (2014). Ingresar a la cultura escrita y a las prácticas sociales del lenguaje, una configuración didáctica para el inicio de la escolaridad. Bogotá: Pontificia Universidad Javeriana.

Reyes, Y. (2008). La biblioteca para los que «no saben leer»: acceso a libros y lecturas en la primera infancia. En R. E. Bonilla, D. Goldin y R. Salaberría (eds.), Bibliotecas y escuelas: retos y posibilidades en la sociedad del conocimiento (pp. 209-238). México, D.F: Océano Travesía.

Reyes, Y. (2007). La casa imaginaria: lectura y literatura en la primera infancia. Bogotá: Norma. Sandoval, C. y Landaverry, R. (2019). Prácticas lectoras en una biblioteca pública de Lima: el caso de los niños lectores asiduos. Revista Educación, 28(54), 105-126.

Sagástegui, C, Stojnic, L y Velásquez, T. (2018). El enfoque de responsabilidad social universitaria (RSU) de la Pontificia Universidad Católica del Perú (PUCP). Lima: DARS.

Sagástegui, C. (2014). Responsabilidad Social Universitaria y aprendizaje por competencias. Percepción de las y los docentes de la PUCP sobre el aprendizaje por competencias en proyectos y cursos vinculados con la RSU. Informe DARS No1. Lima: PUCP.

Vallaeys, F. (2014). La responsabilidad social universitaria: un nuevo modelo universitario contra la mercantilización. Revista Iberoamericana de Educación Superior (RIES), V(12), 105-117. Recuperado de http://ries.universia.net/index.php/ries/article/view/439 